\title{
Study of lattice QCD at finite chemical potential using the canon- ical ensemble approach
}

\author{
V. G. Bornyakov $v^{1,3,4, \star}$, D. L. Boyda ${ }^{1,2,4}$, V. A. Goy ${ }^{1,2,4}$, A. V. Molochkov ${ }^{1,4}$, Atsushi Nakamura ${ }^{1,5,6}$, \\ A. A. Nikolaev ${ }^{1,4}$, and V. I. Zakharov ${ }^{1,4}$ \\ ${ }^{1}$ School of Biomedicine, Far Eastern Federal University, 690950 Vladivostok, Russia \\ ${ }^{2}$ School of Natural Sciences, Far Eastern Federal University, 690950 Vladivostok, Russia \\ ${ }^{3}$ Institute for High Energy Physics NRC Kurchatov Institute, 142281 Protvino, Russia \\ ${ }^{4}$ Institute of Theoretical and Experimental Physics NRC Kurchatov Institute, 117218 Moscow, Russia \\ ${ }^{5}$ Research Center for Nuclear Physics (RCNP), Osaka University, Ibaraki, Osaka, 567-0047, Japan \\ ${ }^{6}$ Theoretical Research Division, Nishina Center, RIKEN, Wako 351-0198, Japan
}

\begin{abstract}
A new approach to the computation of the canonical partition functions in $N_{f}=2$ lattice QCD is presented. The results for the number density as function of imaginary chemical potential obtained in the confining and deconfining phases are used to determine the phase transition line at real chemical potential.
\end{abstract}

\section{Introduction}

Recent results of heavy ion collision experiments at RHIC [1] and LHC [2] shed some light on the properties of the quark gluon plasma and the position of the transition line in the temperature - baryon density plane. New experiments at lower energy will be carried out at FAIR (GSI) and NICA (JINR). To fully explore the phase diagram theoretically it is necessary to make computations at finite temperature and finite baryon chemical potential. For finite temperature, lattice QCD is the only ab-initio method available and many results had been obtained. However, for finite baryon density lattice QCD faces the so-called complex action problem (or sign problem) which prevents direct simulations. Various proposals exist at the moment to solve this problem see, e.g. reviews [3-5], and yet it is still very hard to get reliable results at $\mu_{B} / T>1$. Our work is devoted to the further development of the canonical partition function approach.

The fermion determinant at nonzero baryon chemical potential $\mu_{B}$, det $\Delta\left(\mu_{B}\right)$, is in general not real. This makes it impossible to apply standard importance sampling techniques to systems described by the grand canonical partition function

$$
Z_{G C}\left(\mu_{q}, T, V\right)=\int \mathcal{D} U\left(\operatorname{det} \Delta\left(\mu_{q}\right)\right)^{N_{f}} e^{-S_{G}},
$$

where $S_{G}$ is some gauge field action, $\mu_{q}=\mu_{B} / 3$ is the quark chemical potential, $T=1 /\left(a N_{t}\right)$ is the temperature, $V=\left(a N_{s}\right)^{3}$ is the volume, $a$ is the lattice spacing, and $N_{t}, N_{s}$ - the numbers of lattice sites in time and space directions.

\footnotetext{
^e-mail: vitaly.bornyakov@ihep.ru
} 
The canonical approach was studied in a number of papers [6-13]. It is based on the following relations. The representation of the grand canonical partition function $Z_{G C}\left(\mu_{q}, T, V\right)$ in terms of the canonical ones $Z_{C}(n, T, V)$ (where $\xi=e^{\mu_{q} / T}$ is the fugacity)

$$
Z_{G C}(\mu, T, V)=\sum_{n=-\infty}^{\infty} Z_{C}(n, T, V) \xi^{n},
$$

is called fugacity expansion. The inverse of this equation can be written in the form [14]

$$
Z_{C}(n, T, V)=\int_{0}^{2 \pi} \frac{d \theta}{2 \pi} e^{-i n \theta} Z_{G C}(\theta, T, V) .
$$

$Z_{G C}(\theta, T, V)$ is the grand canonical partition function for imaginary chemical potential $\mu_{q}=i \mu_{q I} \equiv$ $i T \theta$. Standard Monte Carlo simulations are able to represent the measure of this partition function since the fermionic determinant is real for imaginary $\mu_{q}$.

At imaginary chemical potential, the QCD partition function $Z_{G C}$ is a periodic function of $\theta$ : $Z_{G C}(\theta)=Z_{G C}(\theta+2 \pi / 3)$. This symmetry is called Roberge-Weiss symmetry [15]. As a consequence of this periodicity the canonical partition functions $Z_{C}(n, T, V)$ are nonzero only for net quark numbers $n=3 k$. QCD reveals a rich phase structure at nonzero $\theta$, depending on the number of flavors $N_{f}$ and the (degenerate) quark mass $m$. This phase structure is shown in figure $1 . T_{c}$ is the confinement/deconfinement crossover point at zero chemical potential. The line $\left(T \geq T_{R W}, \mu_{I} / T=\pi / 3\right)$ indicates the first of a series of first order phase transitions at $\mu_{I} / T=(2 k+1) \pi / 3$. Along the curve between $T_{c}$ and $T_{R W}$, the crossover is expected to change to a first order transition for small and large quark masses, see e.g. [16]. The quark number density $n_{q}$ for $N_{f}$ degenerate quark flavours is defined

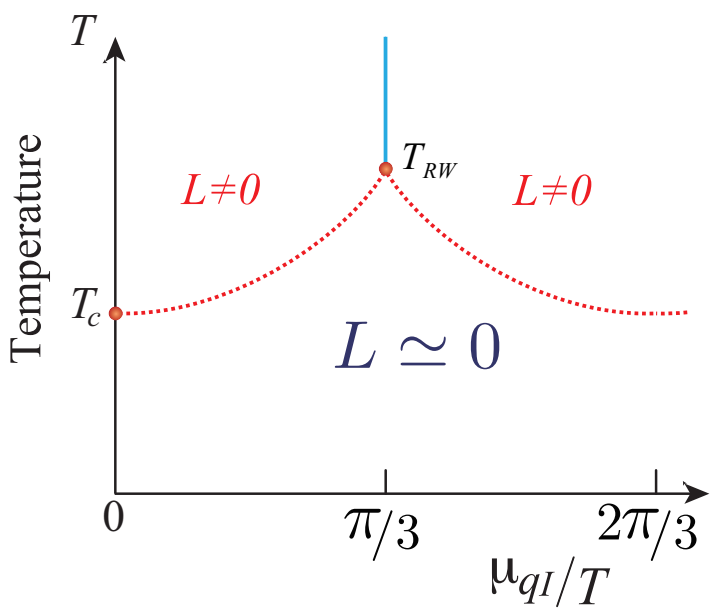

Figure 1. Schematic figure of Roberge-Weiss phase structure in the pure imaginary chemical potential regions.

by the following equation:

$$
\frac{n_{q}}{T^{3}}=\frac{1}{V T^{2}} \frac{\partial}{\partial \mu_{q}} \ln Z_{G C}=\frac{N_{f} N_{t}^{3}}{N_{s}^{3} Z_{G C}} \int \mathcal{D} U e^{-S_{G}}\left(\operatorname{det} \Delta\left(\mu_{q}\right)\right)^{N_{f}} \operatorname{tr}\left[\Delta^{-1} \frac{\partial \Delta}{\partial \mu_{q} / T}\right] .
$$

It can be computed numerically for imaginary chemical potential, and $n_{q}$ turns out also purely imaginary: $n_{q}=i n_{q I}$. 
From (2) and (4) it follows that the densities $n_{q}$ and $n_{q I}$ are related to $Z_{C}(n, T, V)$ (in the following we will use the notation $Z_{n}$ for the ratio $\left.Z_{C}(n, T, V) / Z_{C}(0, T, V)\right)$ by the equations

$$
n_{q} / T^{3}=\mathcal{N} \frac{2 \sum_{n>0} n Z_{n} \sinh (n \theta)}{1+2 \sum_{n>0} Z_{n} \cosh (n \theta)}, n_{q I} / T^{3}=\mathcal{N} \frac{2 \sum_{n>0} n Z_{n} \sin (n \theta)}{1+2 \sum_{n>0} Z_{n} \cos (n \theta)}
$$

$\mathcal{N}=\frac{N_{t}^{3}}{N_{s}^{3}}$ is a normalization constant. Our suggestion is to compute $Z_{n}$ using equation (5) for $n_{q I}$.

One can compute $Z_{G C}(\theta, T, V)$ using numerical data for $n_{q I} / T^{3}$ via numerical integration

$$
L_{Z}(\theta) \equiv \log \frac{Z_{G C}(\theta, T, V)}{Z_{G C}(0, T, V)}=-V \int_{0}^{\theta} d \tilde{\theta} n_{q I}(\tilde{\theta}),
$$

where we have omitted the arguments $T$ and $V$ from the grand canonical partition function for brevity. Then the $Z_{n}$ can be computed as

$$
Z_{n}=\frac{\int_{0}^{2 \pi} \frac{d \theta}{2 \pi} e^{-i n \theta} e^{L_{Z}(\theta)}}{\int_{0}^{2 \pi} \frac{d \theta}{2 \pi} e^{L_{Z}(\theta)}}
$$

In our work we use a modified version of this approach [17]. Instead of performing the numerical integration in (6) we fitted $n_{q I} / T^{3}$ to theoretically motivated functions of $\mu_{q I}$. It is known that the density of a noninteracting quark gas is described by

$$
n_{q} / T^{3}=N_{f}\left(2 \frac{\mu_{q}}{T}+\frac{2}{\pi^{2}}\left(\frac{\mu_{q}}{T}\right)^{3}\right)
$$

We thus fitted the data for $n_{q I}$ to a polynomial containing odd powers of $\theta$

$$
n_{q I}(\theta) / T^{3}=\sum_{n=1}^{n_{\max }} a_{2 n-1} \theta^{2 n-1}
$$

in the deconfining phase. This type of the fit was also used in [18] and [19].

In the confining phase (below $T_{c}$ ) the hadron resonance gas model provides a good description of the chemical potential dependence of thermodynamic observables [20]. Thus, it is reasonable to fit the density in the confining phase by a Fourier expansion

$$
n_{q I}(\theta) / T^{3}=\sum_{n=1}^{n_{\max }} f_{3 n} \sin (3 n \theta)
$$

Again this type of the fit was used in [18], were the conclusion was that it works well.

To demonstrate our method we made simulations of lattice QCD with $N_{f}=2$ clover improved Wilson quarks and Iwasaki improved gauge field action (for detailed definitions of the lattice action see [17]). We have simulated on $16^{3} \times 4$ lattices at temperatures $T / T_{c}=1.35,1.20$ and 1.08 in the deconfinement phase and $T / T_{c}=0.99,0.93,0.84$ in the confinement phase along the line of constant physics with $m_{\pi} / m_{\rho}=0.8$. All parameters of the action, including the $c_{S W}$ parameter, were taken from the WHOT-QCD collaboration paper [21]. We have computed the number density on samples of $N_{\text {conf }}=1800$ configurations, using every 10-th trajectory produced by the Hybrid Monte Carlo algorithm. 


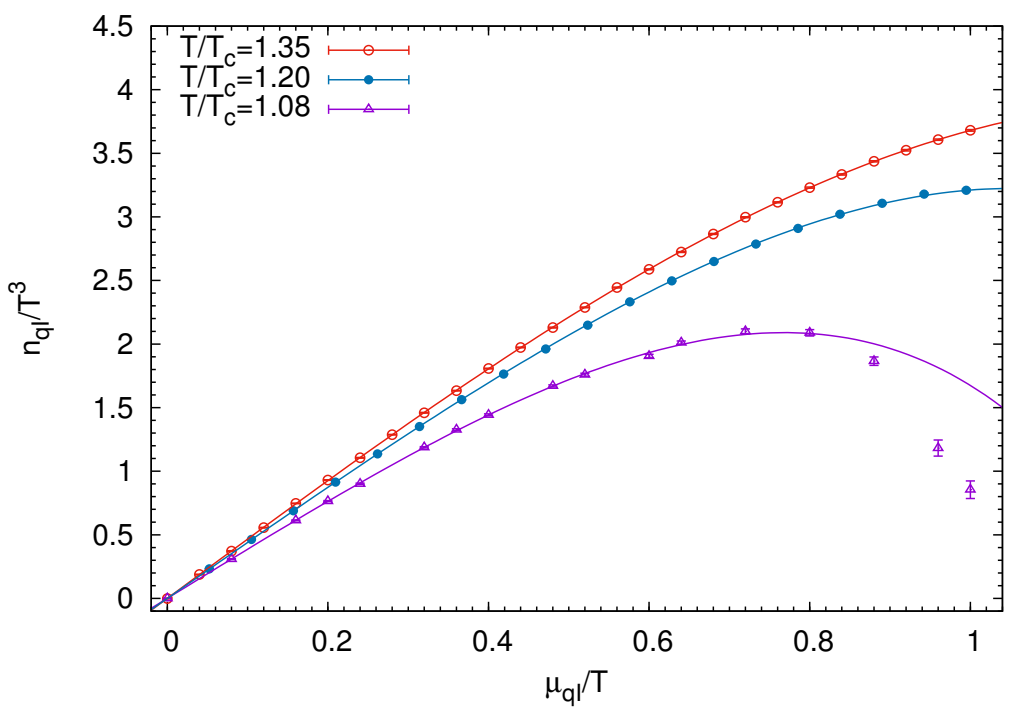

Figure 2. The quark density $n_{q I}$ as function of $\theta$ in the deconfinement phase at temperatures $T / T_{c}=$ $1.35,1.20,1.08$. The curves show fits to the function (9).

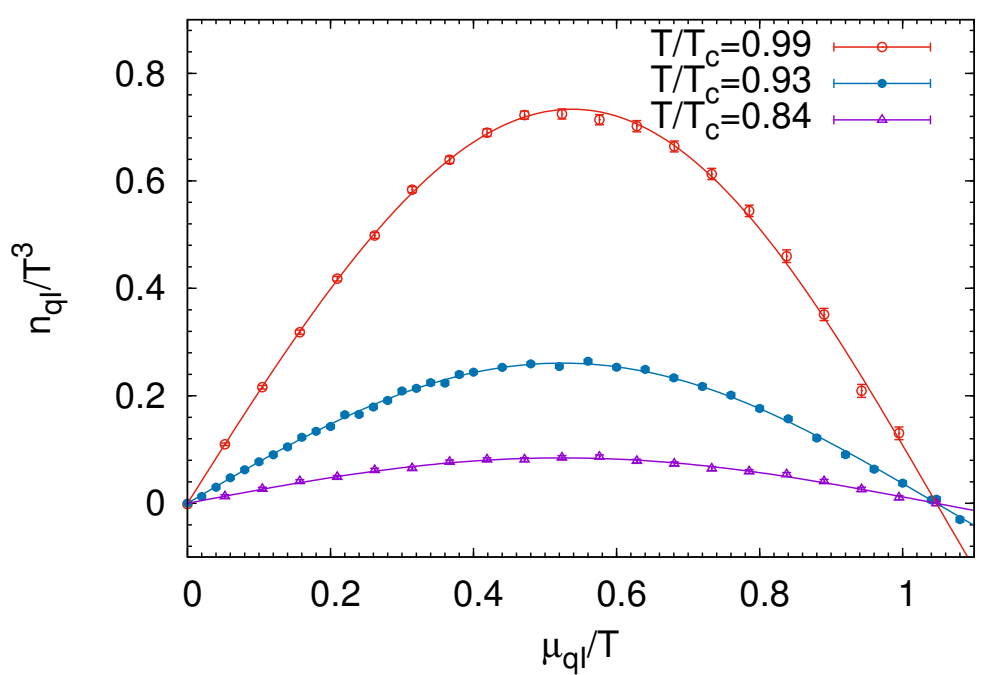

Figure 3. The quark density $n_{q I}$ as function of $\theta$ in the confining phase. The curves show fits to (10) with $n_{\max }=1$ for $T / T_{c}=0.84,0.93$ and $n_{\max }=2$ for $T / T_{c}=0.99$.

\section{Results}

In figure 2 and figure 3 we show numerical results for $n_{q I}$ as a function of $\theta$ in the deconfining and confining phases, respectively [17]. Note, that the behavior of $n_{q I}$ at $T=1.08 T_{c}$ is different from that at higher temperatures. This temperature is still below $T_{R W}$, and at $\theta=\pi / 3$ there is no first order 
phase transition to be hit. Instead, $n_{q I}$ is continuous and passes a crossover to the confinement phase at about $\theta=0.92(2)$.

It is not yet clear how to fit the data over a range of $\mu_{q I}$ connecting pieces of deconfining and confining phase. Here we used a fit to the function (9) with $n_{\max }=3$ over the range $\theta \in[0,0.8]$, i.e. including only the deconfining phase. In this case we should consider the fit as a Taylor expansion.

We have computed $Z_{n}$ using the new procedure described in the previous section and compared it with results obtained with use of the hopping parameter expansion. We found good agreement between two methods indicating that the new method works well [17]. This allows us to proceed with an analytical continuation to the real values of $\mu_{q}$ beyond the Taylor expansion validity range (for all temperatures except $T / T_{c}=1.08$ ). Respective results are shown in figure 4 . Using the

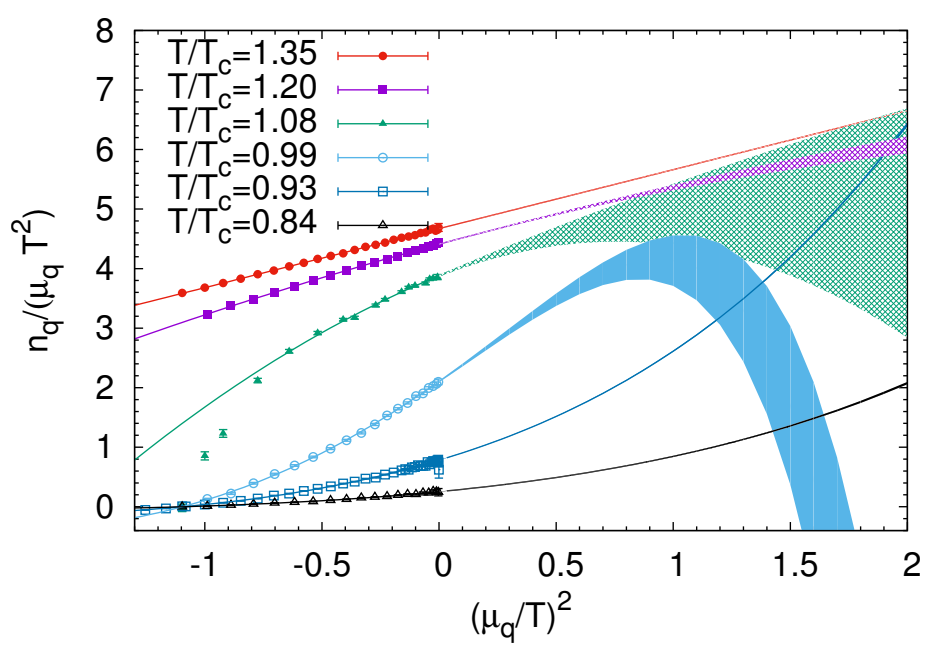

Figure 4. Analytical continuation for the number density vs. $\mu_{q}^{2}$. The curves show respective fits (9) or (10). The width of the curves indicates the statistical error of the extrapolation to $\mu_{q}^{2}>0$.

results for the number density $n_{q}$ we can compute the temperature of the transition from the hadron phase to the quark-gluon plasma phase using the following procedure. Our results for the number density at temperatures $T>T_{c}$ as function of the chemical potential $\mu_{q}$ are reliable even for large $\mu_{q}$ for $T>T_{R W}$, while in the temperature interval $T_{c}<T<T_{R W}$ they are presently reliable for small $\mu_{q}$ only. We can use these results to compute the pressure $\Delta P_{\text {deconf }}\left(T, \mu_{q}\right)=P\left(T, \mu_{q}\right)-P(T, 0)$ as function of $\mu_{q}$ and then extrapolate the pressure for fixed $\mu_{q}$ to temperatures $T<T_{c}$. To obtain a good extrapolation we need the pressure computed for more values of temperature than is available now. But three values which we have in this work are sufficient to demonstrate the idea. We then find the transition temperature $T_{c}\left(\mu_{q}\right)$ solving numerically the equation $\Delta P_{\text {deconf }}\left(T, \mu_{q}\right)=\Delta P_{\text {conf }}\left(T, \mu_{q}\right)$, where $\Delta P_{\text {conf }}\left(T, / m u_{q}\right)$ is the excess pressure obtained from the number density in the confined phase. In this paper we use an extrapolation for the coefficients in (9) rather than for the pressure itself. This extrapolation is shown in figure 5. We fitted the data for $a_{i}, i=1,3,5$ by a polynomial $b_{0}+b_{1} T / T_{c}+$ $b_{2}\left(T / T_{c}\right)^{2}$ and then computed the extrapolated values of these parameters at $T / T_{c}=0.93$ and 0.84 . The extrapolated values were used to compute $\Delta P_{\text {deconf }}\left(T, \mu_{q}\right) / T^{4}$ as

$$
\frac{\Delta P_{\text {deconf }}\left(T, \mu_{q}\right)}{T^{4}}=\frac{a_{1}}{2}\left(\frac{T}{T_{c}}\right)^{2}-\frac{a_{3}}{4}\left(\frac{T}{T_{c}}\right)^{4}+\frac{a_{5}}{6}\left(\frac{T}{T_{c}}\right)^{6}
$$




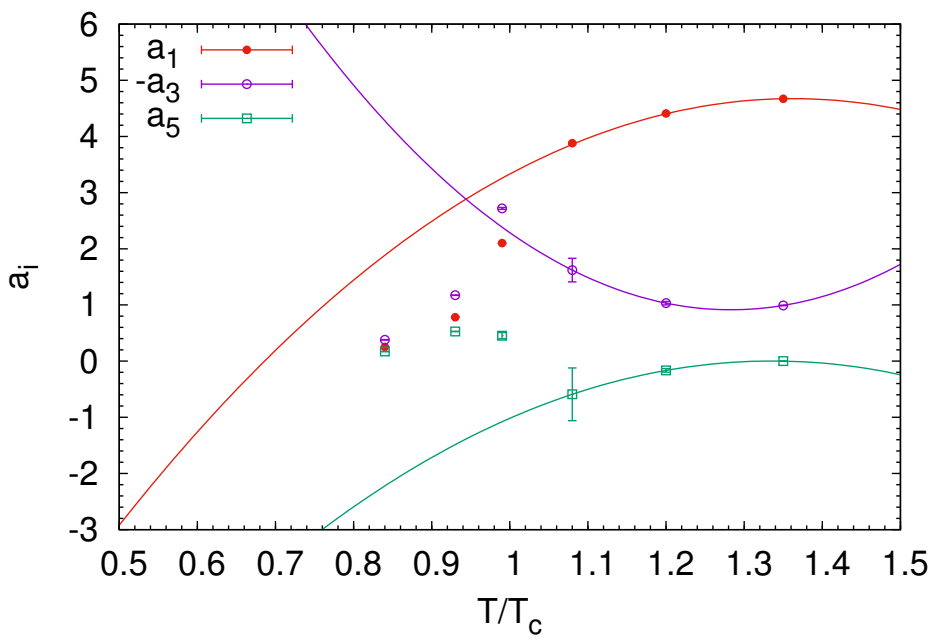

Figure 5. Constants $a_{i}$ of (9) for six values of temperature. The curves show polynomial fits over $T>T_{c}$ range.

We show the results in figure 6 together with the pressure computed by integration of (10) for $T / T_{c}=$

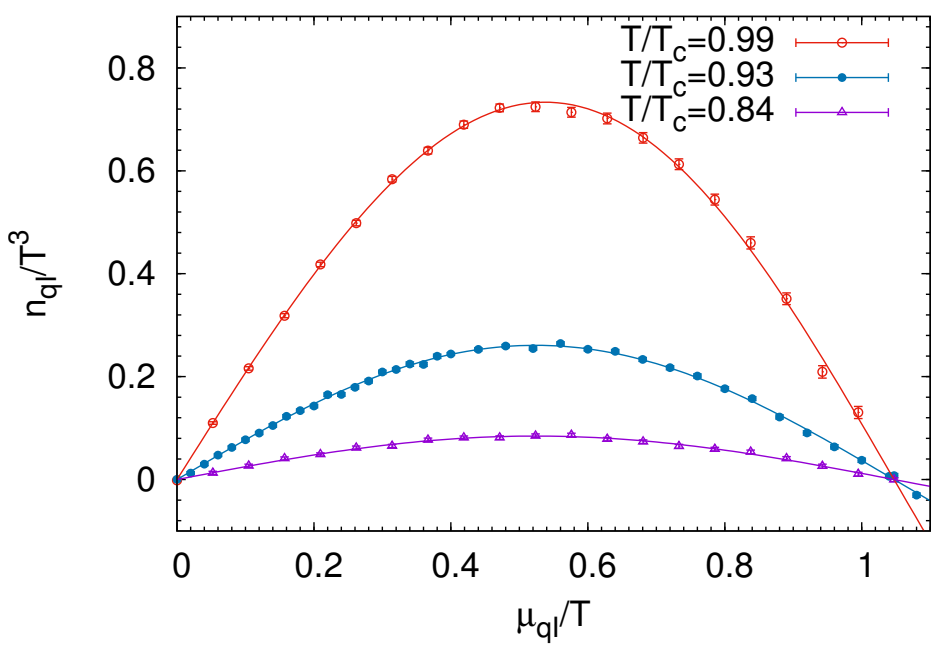

Figure 6. The pressure computed via (11) (denoted by 'deconf', thin curves) and by integration of (10) (denoted by 'conf', thick curves) for $T / T_{c}=0.93,0.84$.

$0.93,0.84$. The crossing points determine the values of $\mu_{q}$ on the transition line at the respective values of temperature. We show the two crossing points in figure 7 together with a fit of the form

$$
T_{c}\left(\mu_{q}\right) / T_{c}=1-C\left(\mu_{q} / T_{c}\right)^{2} .
$$

The result for the fit parameter is $C=0.07$. We have to emphasize that in figures 6 and 7 we do not show errors since we are not yet able to compute them. For the time being, these figures are shown to 


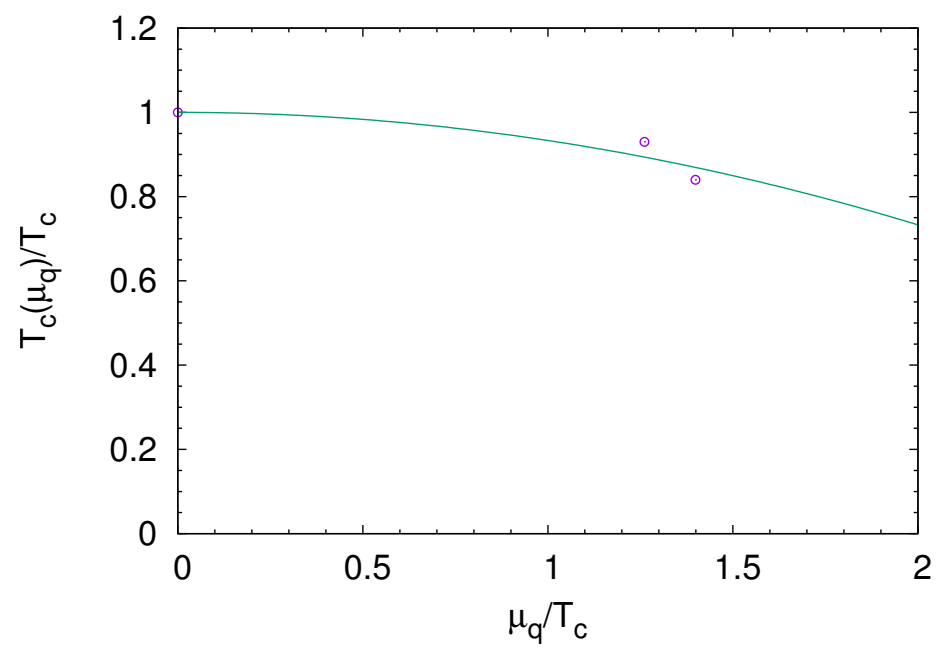

Figure 7. The transition line in the temperature - chemical potential plane. The curve shows a quadratic fit.

illustrate our idea about the method to find the shape of the transition curve. Still it is encouraging that the value of the coefficient $C$ obtained is of correct order of magnitude. We can compare with [22, 23] where $C=0.051(3)$ and $C=0.065(7)$ respectively were found in lattice QCD with $N_{f}=2$. In both papers the direct analytical continuation of $\frac{T_{c}\left(\mu_{q}\right)}{T_{c}}$ from imaginary $\mu_{q}$ was used.

Here we have presented a new method to compute the canonical partition functions $Z_{n}$. It is based on fitting $n_{q I}$ for all values of $\mu_{q I}$ to the theoretically motivated fitting functions: a polynomial fit (9) in the deconfinement phase for $T$ above $T_{R W}$ and a Fourier-type fit (10) in the confinement phase. We have also explained how the transition line at real $\mu_{q}$ can be computed using the results which we obtained for the number density $n_{q}$. We are planning to enlarge the grid of temperature values to improve the method. It is worth to note that the method of direct analytical continuation for $\frac{T_{c}\left(\mu_{q}\right)}{T_{c}}$ from imaginary chemical potential can be applied to our data. This will help us to improve the method presented here.

\section{Acknowledgments}

This work was completed due to support by RSF grant under contract 15-12-20008. Computer simulations were performed on the FEFU GPU cluster Vostok-1 and MSU 'Lomonosov' supercomputer.

\section{References}

[1] J. Adams et al. (STAR), Nucl. Phys. A 757, 102 (2005). nucl-ex/0501009

[2] K. Aamodt et al. (ALICE), JINST 3, S08002 (2008)

[3] S. Muroya, A. Nakamura, C. Nonaka, T. Takaishi, Prog. Theor. Phys. 110, 615 (2003). heplat/0306031

[4] O. Philipsen, PoS (LAT2005) 016 (2006), [PoSJHW2005,012(2006)]. hep-lat/0510077

[5] P. de Forcrand, PoS (LAT2009) 010 (2009). hep-lat/1005.0539

[6] P. de Forcrand, S. Kratochvila, Nucl. Phys. Proc. Suppl. 153, 62 (2006). hep-lat/0602024

[7] S. Ejiri, Phys. Rev. D 78, 074507 (2008). hep-lat/0804.3227

[8] A. Li, A. Alexandru, K.F. Liu, X. Meng, Phys. Rev. D 82, 054502 (2010). hep-lat/1005.4158 
[9] A. Li, A. Alexandru, K.F. Liu, Phys. Rev. D 84, 071503 (2011). hep-ph/1103.3045

[10] J. Danzer, C. Gattringer, Phys. Rev. D 86, 014502 (2012). hep-lat/1204.1020

[11] C. Gattringer, H.P. Schadler, Phys. Rev. D 91, 074511 (2015). hep-lat/1411.5133

[12] R. Fukuda, A. Nakamura, S. Oka, Phys. Rev. D 93, 094508 (2016). hep-lat/1504.06351

[13] A. Nakamura, S. Oka, Y. Taniguchi, JHEP 02, 054 (2016). hep-lat/1504.04471

[14] A. Hasenfratz, D. Toussaint, Nucl. Phys. B 371, 539 (1992)

[15] A. Roberge, N. Weiss, Nucl. Phys. B 275, 734 (1986)

[16] C. Bonati, P. de Forcrand, M. D’Elia, O. Philipsen, F. Sanfilippo, Phys. Rev. D 90, 074030 (2014). hep-lat/1408.5086

[17] V.G. Bornyakov, D.L. Boyda, V.A. Goy, A.V. Molochkov, A. Nakamura, A.A. Nikolaev, V.I. Zakharov (2016), hep-lat/1611.04229

[18] J. Takahashi, H. Kouno, M. Yahiro, Phys. Rev. D 91, 014501 (2015). hep-lat/1410.7518

[19] J. Gunther, R. Bellwied, S. Borsanyi, Z. Fodor, S.D. Katz, A. Pasztor, C. Ratti (2016), heplat/1607.02493

[20] F. Karsch, K. Redlich, A. Tawfik, Phys. Lett. B 571, 67 (2003). hep-ph/0306208

[21] S. Ejiri, Y. Maezawa, N. Ukita, S. Aoki, T. Hatsuda, N. Ishii, K. Kanaya, T. Umeda (WHOTQCD), Phys. Rev. D 82, 014508 (2010). hep-lat/0909.2121

[22] P. de Forcrand, O. Philipsen, Nucl. Phys. B 642, 290 (2002). hep-lat/0205016

[23] L.K. Wu, X.Q. Luo, H.S. Chen, Phys. Rev. D 76, 034505 (2007). hep-lat/0611035 